\title{
Single- and double-stranded DNA: building a trigger of ATR-mediated DNA damage response
}

\author{
Lee Zou ${ }^{1}$ \\ Massachusetts General Hospital Cancer Center and Department of Pathology, Harvard Medical School, \\ Charlestown, Massachusetts 02129, USA
}

The DNA damage signaling pathways mediated by the ataxia-telangiectasia mutated (ATM) and the ATM and Rad3-related (ATR) kinases play crucial roles in the maintenance of genomic integrity and may function as an anti-cancer barrier during early tumorigenesis. Although the ATM and ATR pathways share some of their downstream functions, the DNA damage that evoke these two pathways are distinct. While ATM plays a primary role in the response to double-stranded DNA breaks (DSBs), ATR controls the response to a much broader spectrum of DNA damage, including many that interfere with DNA replication. And, unlike ATM, ATR is crucial for maintaining genomic integrity during $\mathrm{S}$ phase of the cell cycle, and is indispensable for cell survival. Clearly, revealing the DNA structure that elicits the ATR pathway would be a critical step toward understanding the essential function of ATR and the genomic instability that it counters. The versatility of the ATR pathway in DNA damage response suggests that this pathway is likely able to sense a common signal generated by different types of DNA damage and genomic instability. Two simple structures commonly generated at sites of DNA repair and stressed DNA replication forks are single-stranded DNA coated with replication protein A (RPA-ssDNA) and junctions of single- and doublestranded DNA. Both of these structures have been implicated in the activation of ATR checkpoint by a number of studies using different model organisms. In this issue of Genes \& Development, Cimprich and colleagues (MacDougall et al. 2007) report that circular singlestranded DNA (ssDNA) annealed with primers specifically triggers the ATR-mediated checkpoint responses in Xenopus egg extracts, revealing the first defined DNA structure sufficient to activate the ATR checkpoint pathway.

\section{Hints from yeast, Xenopus, and human}

Studies using budding and fission yeast (Saccharomyces cerevisiae and Schizosaccharomyces pombe, respec-

${ }^{1}$ Correspondence.

E-MAIL zou.lee@mgh.harvard.edu; FAX (617) 726-7808.

Article is online at http://www.genesdev.org/cgi/doi/10.1101/gad.1550307. tively) have provided important clues to the DNA structures that activate the ATR checkpoint. Mec1, the S. cerevisiae homolog of ATR, is activated by DNA replication inhibitor hydroxyurea (HU), DNA-alkylating agent methyl methanesulfonate (MMS), ultraviolet light (UV), telomeric defects in the cdc13 mutant, and DSBs induced by the $\mathrm{HO}$ endonuclease. At both the telomeres in the $c d c 13$ mutant and the HO-induced DSBs, ssDNA is generated through the action of $5^{\prime}$-to-3' exonucleases (Garvik et al. 1995; Lee et al. 1998). Accompanying the formation of ssDNA, 3' ssDNA ends and 5' double/ single-stranded DNA (ds/ssDNA) junctions are also generated. It is not clear whether additional DNA structures are formed at these DNA damage sites. The ability of these two specific types of DNA damage to elicit the Mec1-mediated checkpoint indicates that ssDNA and/or ds/ssDNA junctions may be part of the checkpointactivating DNA structure.

DNA replication interference is also a major stimulus of the ATR checkpoint. In budding yeast, electron microscopy studies of stressed replication forks have revealed some of the DNA structures that may contribute to checkpoint activation. In undamaged cells, short ssDNA regions ( 220 nucleotides [nt]) were observed on one side of the replication forks (Sogo et al. 2002). Upon HU treatment, the progression of replication forks was drastically reduced, and the size of ssDNA regions at the forks was increased by $>100 \mathrm{nt}$. In the presence of UVinduced DNA lesions, ssDNA gaps were detected on both leading and lagging strands of the forks (Lopes et al. 2006). The ssDNA gaps on the leading strand were remarkably long (up to $3 \mathrm{~kb}$ ), and those on the lagging strand were shorter $(<400 \mathrm{nt})$. The formation of long stretches of ssDNA on the leading strand suggests that synthesis of this strand was blocked by UV lesions, resulting in uncoupling of leading and lagging strand synthesis. Beside the ssDNA gaps at the forks, small ssDNA gaps (<400 nt) were also found far behind the forks on both strands. Taken together, these studies demonstrated that increased amounts of ssDNA were generated at or behind stressed replication forks. Furthermore, depending on the location of the ssDNA gaps, they can be flanked by $5^{\prime}$ and/or 3' ds/ssDNA junctions. 
In vitro studies using Xenopus egg extracts have also revealed important clues to the DNA structures that activate the ATR checkpoint. Addition of MMS- or UVtreated chromatin, or aphidicolin (an inhibitor of DNA polymerase $\alpha$ ) to Xenopus egg extracts activates the ATR checkpoint in a DNA replication-dependent manner (Lupardus et al. 2002; Stokes et al. 2002). Under these conditions, checkpoint activation relies on the initiation of DNA replication from the prereplication complex (pre-RC). Concomitant with checkpoint activation, increasing amounts of ssDNA-binding protein complex RPA and DNA polymerase $\alpha(\operatorname{Pol} \alpha)$ are recruited onto chromatin (Michael et al. 2000; Lupardus et al. 2002). The recruitment of RPA and Pol $\alpha$ to chromatin, as well as checkpoint activation, require the uncoupling between MCM helicase and DNA polymearase (Pacek and Walter 2004; Byun et al. 2005). Depletion of RPA from extracts prevents the loading of Pol $\alpha$ to chromatin (Lee et al. 2003). Depletion or complete inhibition of $\mathrm{Pol} \alpha$ also prevents checkpoint activation (Michael et al. 2000; You et al. 2002; Byun et al. 2005). These findings are consistent with a model in which the generation of ds/ ssDNA junctions by $\mathrm{Pol} \alpha$ is required for checkpoint activation. Furthermore, chromatin treated with topoisomerase II inhibitor etoposide or Exo III can elicit the ATR checkpoint in a pre-RC-independent, but RPA-dependent manner (Costanzo et al. 2003). These results imply that certain RPA-associated DNA intermediates, such as ssDNA gaps, are able to activate the ATR checkpoint.

In vitro characterizations of purified human, yeast, and Xenopus checkpoint proteins have brought us even closer to the identification of the ATR checkpoint-activating DNA structure. Purified human and yeast ATRIP, the regulatory partner of ATR, directly binds to RPAssDNA and targets the ATR-ATRIP complex to this damage-induced structure (Zou and Elledge 2003). Xenopus ATRIP also recognizes ssDNA in an RPA-dependent manner in extracts (Kim et al. 2005). Two other important regulators of the ATR pathway, the replication factor C (RFC)-like Rad17 complex and the PCNA-like Rad9-Rad1-Hus1 (9-1-1) complex, recognize ds/ssDNA junctions (Bermudez et al. 2003; Ellison and Stillman 2003; Majka and Burgers 2003; Zou et al. 2003). Through a mechanism resembling the loading of PCNA by RFC during DNA replication, the Rad17 complex recruits 9-1-1 complexes onto DNA structures with ds/ssDNA junctions in an RPA-stimulated manner (Ellison and Stillman 2003; Zou et al. 2003; Majka et al. 2006a). Using biotinylated linear ssDNA annealed with primers, it was shown that the $S$. cerevisiae homolog of 9-1-1 complex, when loaded onto DNA, stimulated the kinase activity of Mec1 (Majka et al. 2006b). Thus, it is likely that RPAssDNA and ds/ssDNA junctions are directly involved in the recruitment of ATR-ATRIP and its key regulators to sites of DNA damage, and are sufficient for stimulating the Mecl kinase to some extent. Despite this strong evidence, whether ssDNA and ds/ssDNA junctions are sufficient to induce robust signaling through the ATR checkpoint pathway remained untested.

\section{Defining the ATR checkpoint-activating DNA structure}

An important step toward revealing the ATR checkpoint-activating DNA structure was the discovery that annealed poly $(\mathrm{dA})_{70}$ and poly $(\mathrm{dT})_{70}$, but not $(\mathrm{dA})_{70}$ alone, can induce robust ATR-mediated DNA damage responses in Xenopus egg extracts (Kumagai and Dunphy 2000). A hallmark of activated ATR pathway is the phosphorylation of Chk1, an effector kinase of ATR. Annealed $(\mathrm{dA})_{70}-(\mathrm{dT})_{70}$ induces Chk1 phosphorylation in the absence of replication inhibitors or DNA-damaging agents, suggesting that the ATR checkpoint can be activated by synthetic DNA structures mimicking DNA replication or repair intermediates. The DNA structures formed by annealed $(\mathrm{dA})_{70}-(\mathrm{dT})_{70}$ are heterogeneous. These structures include dsDNA with blunt ends, various partially single-stranded DNA structures, fork-like structures, cruciforms, and very likely long-branched molecules. As a result of this heterogeneity, $(\mathrm{dA})_{70^{-}}$ $(\mathrm{dT})_{70}$ activates not only the ATR but also the ATM responses in extracts (Yoo et al. 2004). Like the DNA damage or stressed replication forks embedded in chromatin, $(\mathrm{dA})_{70}-(\mathrm{dT})_{70}$ recruits ATR-ATRIP in an RPA-dependent manner, and it induces Chk1 phosphorylation in an ATR- and ATRIP-dependent manner (Kumagai et al. 2004). The ATR associated with $(\mathrm{dA})_{70}-(\mathrm{dT})_{70}$ exhibited higher kinase activity than did that bound to ssDNA, indicating that ATR-ATRIP is stimulated on $(\mathrm{dA})_{70}-(\mathrm{dT})_{70}$ (Kumagai et al. 2004). However, unlike chromatin, $(\mathrm{dA})_{70}-(\mathrm{dT})_{70}$ can induce Chk1 phosphorylation even in RPA-depleted extracts where ATR-ATRIP cannot stably associate with DNA (Kim et al. 2005). The conserved coiled-coil domain of ATRIP, which is important for the localization of ATR-ATRIP to DNA damage and Chk1 phosphorylation in human cells (Ball and Cortez 2005; Itakura et al. 2005), is also dispensable for the Chk1 phosphorylation induced by $(\mathrm{dA})_{70}-(\mathrm{dT})_{70}$. Thus, it is clear that certain synthetic DNA structures formed by $(\mathrm{dA})_{70}$ and $(\mathrm{dT})_{70}$ are sufficient to activate the ATR checkpoint. However, what those structures are and whether they recapitulate the structures normally recognized in vivo is unknown. Furthermore, some of the regulatory mechanisms that normally operate on chromatin-localized DNA damage may not be fully recapitulated in this assay.

Is partially single-stranded DNA the structure in the heterogeneous $(\mathrm{dA})_{70}-(\mathrm{dT})_{70}$ mixture that activates the ATR checkpoint? In an earlier study, it was shown that single-stranded M13 DNA annealed with primers did not induce a Chk1 mobility shift (indicative of Chk1 phosphorylation) in nucleoplasmic extracts (NPE) (Stokes et al. 2002). Using a phospho-specific antibody against a particular ATR phosphorylation site of Chk1, Cimprich and colleagues (MacDougall et al. 2007) revisited this question. Surprisingly, they found that primed M13 ssDNA induced robust Chk1 phosphorylation in NPE. Furthermore, they showed that primed M13 ssDNA efficiently inhibited the replication of double-stranded plasmids in the same extracts, confirming the induction 
of a bona fide checkpoint response. The Chk1 phosphorylation induced by primed M13 ssDNA required the presence of free ds/ssDNA junctions but not de novo DNA synthesis. Ongoing DNA synthesis, although not essential, also contributed to Chk1 phosphorylation. Like DNA damage on chromatin, primed M13 ssDNA induced Chk1 phosphorylation through a mechanism dependent upon ATRIP as well as the other known regulators of the ATR pathway, including RPA, Rad1, TopBP1, and Claspin. Importantly, unlike $(\mathrm{dA})_{70}-(\mathrm{dT})_{70}$, primed M13 ssDNA activated the ATR but not the ATM pathway, providing a way to specifically analyze the ATR checkpoint in Xenopus extracts with a defined DNA structure.

Beside the DNA structures involved, the checkpoint assays using $(\mathrm{dA})_{70}-(\mathrm{dT})_{70}$ and primed M13 ssDNA have several other differences (Kumagai and Dunphy 2000; MacDougall et al. 2007). First, two different types of extracts were used. The $(\mathrm{dA})_{70}-(\mathrm{dT})_{70}$ assay used interphase Xenopus egg extracts, whereas the primed M13 ssDNA assay used NPE. Second, the phosphorylation of Chk1 was analyzed differently. The $(\mathrm{dA})_{70}-(\mathrm{dT})_{70}$ assay monitored the mobility shift of in vitro-translated Chk1 on protein gels, whereas the primed M13 ssDNA assay monitored the phosphorylation of endogenous Chk1 with a phospho-specific antibody. Third, tautomycin, a phosphatase inhibitor, is necessary for the detection of phosphorylated Chk1 in the $(\mathrm{dA})_{70}-(\mathrm{dT})_{70}$ assay, but not in the primed M13 ssDNA assay. Inhibition of PP2A led to elevated levels of phosphorylated Chk1 in undamaged human cells (Leung-Pineda et al. 2006). Whether and how tautomycin affects signaling in extracts is unknown. Fourth, $(\mathrm{dA})_{70}-(\mathrm{dT})_{70}$ was typically used at a higher concentration $(50 \mu \mathrm{g} / \mathrm{mL})$ than was primed M13 ssDNA (at little as $1-2 \mu \mathrm{g} / \mathrm{mL}$ ). Since M13 ssDNA ( $>6400$ bases) is much longer than $(\mathrm{dA})_{70}$ and $(\mathrm{dT})_{70}$, on a molar basis, this represents up to 4500 -fold more $(\mathrm{dA})_{70}$ and $(\mathrm{dT})_{70}$ molecules than primed M13 molecules. Interestingly, it is noticeable that primed M13 ssDNA, but not $(\mathrm{dA})_{70}-(\mathrm{dT})_{70}$, induced Chk1 phosphorylation in an RPA-dependent manner. As RPA is required for signaling in vivo and in chromatin-based in vitro assays (Costanzo et al. 2003; Wang and Qin 2003; Zou and Elledge 2003; Andreassen et al. 2004), it raises the question as to whether certain physiological regulation of the ATR pathway is bypassed by high concentrations of $(\mathrm{dA})_{70^{-}}$ $(\mathrm{dT})_{70}$. Additional studies are needed to understand how the differences in the assay conditions affect the signaling of ATR pathway in Xenopus extracts.

\section{The functions of ssDNA and ds/ssDNA junctions}

How does primed M13 ssDNA activate the ATR checkpoint? Primed M13 ssDNA has three basic structural components: ssDNA, dsDNA, and ds/ssDNA junctions. Both ssDNA and ds/ssDNA junctions have been implicated in checkpoint activation, but whether dsDNA has a role separate from forming ds/ssDNA junctions is not known. Since primed M13 ssDNA activates the checkpoint in an RPA-dependent manner, the ssDNA region of primed M13 is probably coated by RPA in extracts and functions as RPA-ssDNA. RPA-ssDNA has several known roles in ATR checkpoint activation. The direct association between ATRIP and RPA-ssDNA enables ATR-ATRIP to recognize this damage-induced structure in vitro, and is required for the accumulation of ATRATRIP at sites of DNA damage in cells (Costanzo et al. 2003; Zou and Elledge 2003; Kumagai et al. 2004; Lucca et al. 2004; Nakada et al. 2004; Ball et al. 2005; Namiki and Zou 2006). In addition to ATRIP, RPA may also interact with ATR in the ATR-ATRIP complex to stabilize its association with RPA-ssDNA (Nakada et al. 2005). The recruitment of ATR-ATRIP to RPA-ssDNA may be involved in directing the ATR-ATRIP kinase to its substrates, stimulating its kinase activity, retaining the kinase at sites of DNA damage, amplifying DNA damage signals, or combinations of these functions. Like many other RPA-interacting proteins, ATRIP associates with RPA-ssDNA through multiple interactions (Namiki and Zou 2006). One of the RPA-ssDNA-interacting domains of ATRIP was mapped to its $\mathrm{N}$ terminus (Ball et al. 2005; Namiki and Zou 2006). This domain is important for the accumulation of ATR-ATRIP at sites of DNA damage, but not essential for Chk1 phosphorylation (Ball et al. 2005). How the other ATRIP-RPA-ssDNA interactions contribute to DNA damage signaling in cells remains to be determined. Like ATR-ATRIP, the Tipin-Timeless complex, which is required for the efficient phosphorylation of Chk1 by ATR-ATRIP, also interacts with RPAssDNA (Gotter et al. 2007; Unsal-Kacmaz et al. 2007). These findings suggest that part of the DNA damage signaling complex is assembled on RPA-ssDNA.

Although RPA-ssDNA is an important component of the checkpoint-activating structure, it is not sufficient to activate the checkpoint. In the presence of ds/ssDNA junctions, RPA-ssDNA plays two additional roles: It helps to recruit the Rad17 complex to damaged DNA, and it enables the Rad17 complex to load 9-1-1 complexes onto DNA (Ellison and Stillman 2003; Zou et al. 2003). An interesting finding by Cimprich and colleagues (MacDougall et al. 2007) is that $5^{\prime}$ ds/ssDNA junctions activate the checkpoint more efficiently than $3^{\prime} \mathrm{ds} /$ ssDNA junctions when replication is blocked by aphidicolin. This result raises at least three possibilities. First, the Rad17 complex recruits 9-1-1 complexes more efficiently to $5^{\prime} \mathrm{ds} / \mathrm{ssDNA}$ junctions than to $3^{\prime} \mathrm{ds} / \mathrm{ssDNA}$ junctions. This would be consistent with two in vitro studies; one using purified human Rad17 and 9-1-1 complexes (Ellison and Stillman 2003), and the other using their yeast homologs (Majka et al. 2006a). Second, the DNA polymerase inhibited by aphidicolin may prevent the Rad17 complex from recognizing 3' ds/ssDNA junctions. Third, the binding of Rad17 complex to $3^{\prime}$ ds/ ssDNA junctions may be inhibited by other factors in the extract. For example, RFC, other RFC-like complexes, or various DNA polymerases may compete with the Rad17 complex for 3' ds/ssDNA junctions.

It is interesting to consider how $5^{\prime}$ ds/ssDNA junctions are generated at stressed DNA replication forks. When DNA synthesis on the leading strand is hindered, 
replication may restart ahead of the blocked polymerase, leaving ssDNA gaps and 5' ds/ssDNA junctions behind the forks (Lopes et al. 2006). If DNA synthesis on the lagging strand is interrupted, persistent ssDNA gaps and 5' ds/ssDNA junctions may be generated between Okazaki fragments. It should be noted that the Pol $\alpha$-primase complexes at replication forks generate $5^{\prime}$ RNA/DNA junctions instead of $5^{\prime} \mathrm{ds} / \mathrm{ssDNA}$ junctions. When tested in the primed M13 assay, RNA primers were rapidly degraded in NPE and failed to activate the checkpoint. Thus, it remains to be determined whether the processing of $5^{\prime}$ RNA/DNA junctions to $5^{\prime}$ ds/ssDNA junctions is necessary for checkpoint activation at the forks. Nevertheless, the results by Cimprich and colleagues (MacDougall et al. 2007) demonstrate that 5' ds/ssDNA junctions or their derivatives, such as those generated at DSBs, can activate the checkpoint in the absence of an RNA component.

Cimprich and colleagues (MacDougall et al. 2007) also showed that $3^{\prime}$ ds/ssDNA junctions, although less efficient than $5^{\prime}$ junctions, activated the checkpoint to some extent in the absence of DNA synthesis. When present in the replicating state, $3^{\prime} \mathrm{ds} / \mathrm{ssDNA}$ junctions elicited the checkpoint more efficiently. These findings suggest that $3^{\prime}$ ds/ssDNA junctions also contribute to checkpoint activation, and that their function is influenced by the protein factors, DNA structures, and/or other dynamic changes associated with DNA synthesis. Consistent with this idea, a previous in vitro study showed that the Rad17 complex can function at both $5^{\prime}$ and $3^{\prime} \mathrm{ds} /$ ssDNA junctions (Zou et al. 2003). Together, these results imply that the $3^{\prime} \mathrm{ds} / \mathrm{ssDNA}$ junctions on both the leading and lagging strands may contribute to checkpoint activation.

How is the ATR checkpoint activated when both ATR-ATRIP and 9-1-1 are recruited to primed M13 ssDNA? A recent biochemical study using purified yeast checkpoint complexes has provided a clue to this question. When loaded onto partially single-stranded DNA, the yeast homolog of 9-1-1 (Rad17-Mec3-Ddc1) enhanced the kinase activity of Mec1-Ddc2(ATR-ATRIP) (Majka et al. 2006b). Whether human and Xenopus 9-1-1 complexes can stimulate ATR-ATRIP kinase is still unknown. Even if 9-1-1 stimulates ATR-ATRIP, it is not the only factor that can do so. Xenopus TopBP1, an important regulator of the ATR checkpoint, can also stimulate the kinase activity of ATR-ATRIP, even in the absence of any DNA (Hashimoto et al. 2006; Kumagai et al. 2006). In budding yeast, the Ddc1(Rad9) protein, a component of the 9-1-1 homolog, binds to Dpb11, the TopBP1 ortholog (Wang and Elledge 2002). In fission yeast, this interaction with $\operatorname{Rad} 4(\mathrm{TopBP} 1)$ also occurs and was shown to be dependent upon a Rad3(ATR) phosphorylation site on Rad9. This interaction between Rad9 and $\operatorname{Rad} 4(\mathrm{TopBP} 1)$ is required for checkpoint activation (Furuya et al. 2004), raising the possibility that 9-1-1 may bring TopBP1 to ATR-ATRIP after ATR phosphorylation of Rad9. Whether this proposed mechanism operates in Xenopus extracts or human cells is unclear (St Onge et al. 2003; Lupardus and Cimprich 2006). While
9-1-1 and TopBP1 are important regulators of the ATRATRIP kinase, they are not required for some of the early events during the DNA damage response. In both budding and fission yeast, neither 9-1-1 nor TopBP1 homologs are needed for the damage-induced phosphorylation of ATRIP homologs (Edwards et al. 1999; Paciotti et al. 2000). In mammalian cells, the HU-induced phosphorylation of histone $\mathrm{H} 2 \mathrm{AX}$ is ATR dependent, but 9-1-1 independent (Ward and Chen 2001). Moreover, the damage-induced phosphorylation of Xenopus TopBP1 is required for TopBP1 to stimulate ATR-ATRIP, suggesting that TopBP1 functions to amplify DNA damage signals (Hashimoto et al. 2006). How exactly the DNA damage signals are generated by the checkpoint proteins is still unclear, and the defined checkpoint-activating structures like primed M13 ssDNA will help to address this important question.

\section{Quantitative control of DNA damage signals}

If primed ssDNA is the basic structure that activates the ATR checkpoint, then how many of these structures are needed in cells to activate the checkpoint? Through a careful titration of primed M13 ssDNA in the checkpoint assay, Cimprich and colleagues (MacDougall et al. 2007) estimated that in each somatic cell nucleus 30 ds/ssDNA junctions are sufficient to induce detectable levels of Chk1 phosphorylation. However, the number of ds/ssDNA junctions is not the only factor that controls ATR signaling quantitatively. Using M13 ssDNA annealed with two biotinylated primers, Cimprich and colleagues (MacDougall et al. 2007) found that the length of ssDNA adjacent to ds/ssDNA junctions is another important determinant of the strength of DNA damage signals. Indeed, at HO-induced DSBs in yeast, the formation of long stretches of ssDNA is needed for robust phosphorylation of Rad53, an effector kinase of Mec1 (Vaze et al. 2002). Given that primed M13 ssDNA activates the checkpoint in an RPA-dependent manner, it is plausible that the amounts of RPA-ssDNA adjacent to ds/ssDNA junctions determine how potent these junctions are for checkpoint activation. It is interesting to note that although unrepaired UV lesions induced long ssDNA at replication forks (Lopes et al. 2006), the increase of ssDNA at each HU-stressed fork was relatively moderate (Sogo et al. 2002). The ability of HU to efficiently activate the checkpoint may rely on the increase of ssDNA at a large number of stressed forks. Alternatively, there may be factors other than ssDNA that can regulate HUinduced signals quantitatively.

How is the length of RPA-ssDNA sensed by the checkpoint? Purified ATRIP is more efficiently recruited to long RPA-ssDNA compared with short RPA-ssDNA (Zou and Elledge 2003), suggesting that the ATR-ATRIP kinase itself may be a quantitative sensor of RPAssDNA. Consistent with this, the accumulation of Mec1 at $\mathrm{HO}$-induced DSBs in yeast requires RPA as well as substantial amounts of ssDNA (Nakada et al. 2004). Interestingly, the yeast $x r s 2 \Delta$ exo1s mutant defective in 
generating long ssDNA at the HO-induced DSBs was unable to efficiently recruit $\mathrm{Mec1}$, but was able to recruit Ddc1 (a component of the yeast 9-1-1 homolog), to the DSBs (Nakada et al. 2004). These results suggest that the recruitment of 9-1-1, unlike that of ATR-ATRIP, is not regulated by RPA-ssDNA in a length-dependent manner. The RPA-ssDNA of primed M13 may function to enrich the ATR-ATRIP kinase on DNA, allowing it to be efficiently stimulated by regulators. Long RPAssDNA may also help to recruit regulators and substrates of ATR-ATRIP as well as downstream signaling proteins such as the Tipin-Timeless complex. The RPA-ssDNAmediated interactions among the checkpoint proteins may enable them to transmit and amplify signals efficiently.

\section{A multistep model for ATR checkpoint activation}

From the results of recent in vivo and in vitro studies, a multistep model for ATR checkpoint activation has emerged (Fig. 1). The full activation of the ATR pathway requires not only the generation of initial DNA damage signals, but also the amplification of these signals. The regulatory mechanisms involved in the early steps of this pathway likely operate on DNA damageinduced structures such as primed ssDNA, whereas some of the signal amplifying mechanisms may operate off DNA.

At the beginning of the activation process is the generation of ssDNA and ds/ssDNA junctions at stressed replication forks and sites of DNA damage. Once coated by RPA, primed ssDNA recruits and enriches ATRATRIP, Rad17, and 9-1-1 complexes at these sites (Zou and Elledge 2003; Zou et al. 2003). Additional signaling proteins such as Tipin and Timeless may also be recruited by RPA-ssDNA. During this stage, ATRIP becomes phosphorylated by ATR even in the absence of Rad17 and 9-1-1. How the phosphorylation of ATRIP is regulated by DNA damage, and whether and how it contributes to signaling, are currently unknown (Itakura et al. 2004). The colocalization of ATR-ATRIP, Rad17, and 9-1-1 complexes on damaged DNA may provide a mechanism to up-regulate the kinase activity of ATRATRIP and to bring about the phosphorylation of a subset of ATR substrates including Rad17 and Rad9. Phosphorylated Rad17 and Rad9 may function to recruit downstream signaling proteins such as Claspin and TopBP1 (St Onge et al. 2003; Wang et al. 2006), both of which are also phosphorylated by ATR-ATRIP. Once phosphorylated, TopBP1 is transformed into a potent stimulator of ATR-ATRIP, and it may trigger a "forward-feeding" loop of signaling (Hashimoto et al. 2006; Kumagai et al. 2006). The phosphorylation of Claspin, on the other hand, may enable it to facilitate the phosphorylation and activation of Chk1 through stable proteinprotein interaction (Kumagai and Dunphy 2003; Osborn and Elledge 2003; Zhao et al. 2003; Kumagai et al. 2004; Chini and Chen 2006; Yoo et al. 2006). Although this model unifies many recent findings on the ATR checkpoint pathway, it may not represent a comprehensive

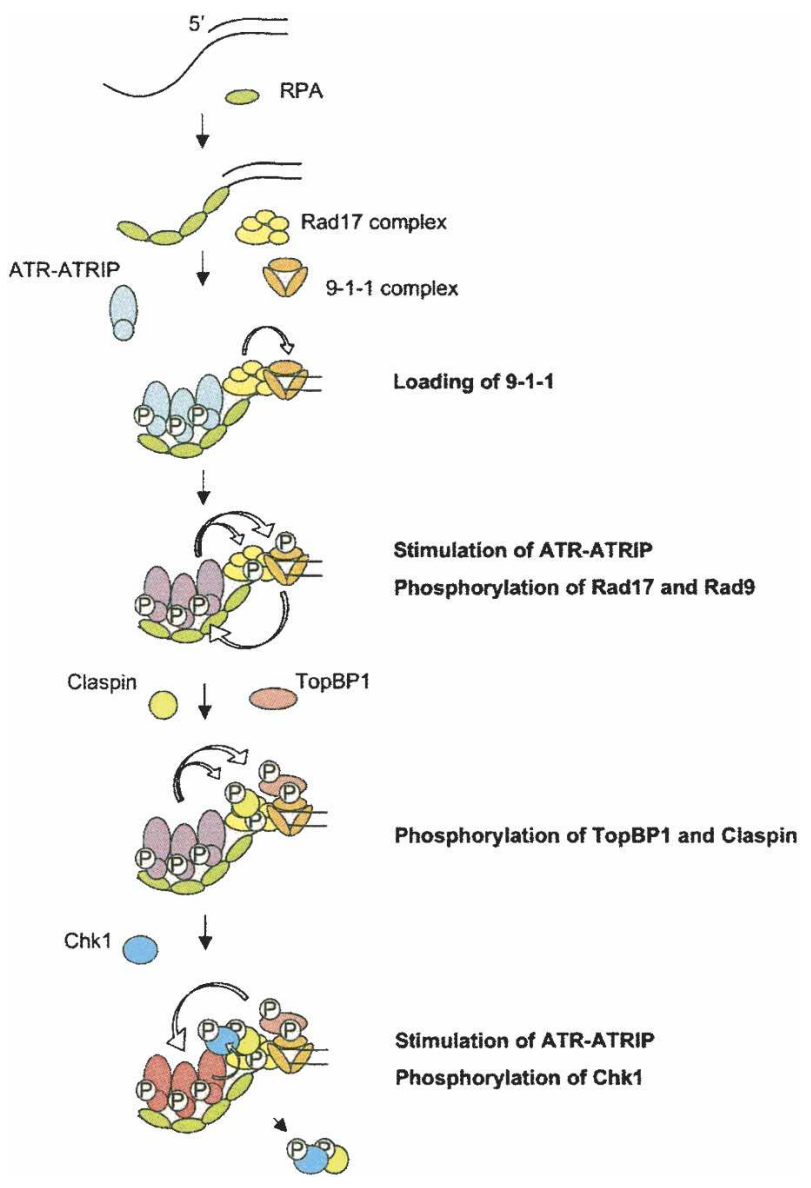

Figure 1. A simplified model for the activation of ATR checkpoint by primed ssDNA. RPA-coated ssDNA recruits ATRATRIP and facilitates the loading of $9-1-1$ to ds/ssDNA junctions by the Rad17 complex. The 9-1-1 complexes on DNA may then stimulate the kinase activity of ATR-ATRIP, enabling it to phosphorylate substrates including Rad17 and Rad9. Phosphorylated Rad17 and Rad9 may facilitate the recruitment of Claspin and TopBP1, respectively, allowing them to be efficiently phosphorylated by ATR. Phosphorylated TopBP1 may further stimulate the kinase activity of ATR-ATRIP. The phosphorylation of Claspin by ATR may promote its interaction with Chk1, which facilitates the phosphorylation of Chk1 by ATR-ATRIP.

understanding of the pathway. It should be emphasized that many of the regulatory mechanisms in this model may not operate in a simple linear fashion. Understanding how the different regulatory mechanisms of the ATR pathway operate in concert requires further investigations.

\section{Beyond the minimal DNA structure for checkpoint activation}

While many proteins involved in DNA damage signaling have been identified, in only a few cases has their regulation by DNA damage been even partially understood. The minimal DNA structure required for ATR check- 
point activation will facilitate the biochemical characterization of the checkpoint proteins, providing insights into how DNA damage signals are generated and transmitted by these proteins. In addition, as demonstrated by Cimprich and colleagues (MacDougall et al. 2007), the checkpoint assay that they developed can also be used to further characterize the DNA structures involved in DNA damage signaling. Even though primed ssDNA is sufficient to activate the ATR checkpoint, additional DNA structures and proteins at stressed replication forks or sites of DNA repair may provide further regulation on DNA damage signaling. It is interesting to point out that although many types of DNA damage can activate the ATR checkpoint, the signaling process evoked by these different types of DNA damage may be regulated differently. For instance, phosphorylation of Rad17 and Claspin plays different roles in response to different types of DNA damage (Wang et al. 2006; Yoo et al. 2006). Moreover, the DSB-induced ATR signaling, but not that induced by replication stress, is regulated by ATM and Mre11 (Cuadrado et al. 2006; Jazayeri et al. 2006; Myers and Cortez 2006). Together with in vivo approaches, the in vitro checkpoint assay described here may help us understand how the ATR pathway is regulated by different types of DNA damage.

As with other valuable in vitro assays, the checkpoint assay using primed M13 ssDNA may not recapitulate all the signaling mechanisms that operate in vivo. For example, chromatin modifications such as phosphorylation and methylation of histones are involved in the DSB-induced checkpoint signaling in humans and yeast. How primed ssDNA activates the ATR pathway in the context of chromatin remains to be tested. Furthermore, in addition to Chk1 phosphorylation and inhibition of DNA synthesis, the ATR pathway mediates many other types of local or cell-wide DNA damage responses in vivo, some of which may not be supported by primed M13 ssDNA. A comprehensive understanding of how the ATR pathway is regulated by DNA damage will require combined in vivo and in vitro approaches using multiple model organisms.

\section{Acknowledgments}

I thank Drs. Stephen Elledge, Karlene Cimprich, Lei Li, and members of the Zou laboratory for helpful discussions. Research of my laboratory is supported by grants from NIH (GM076388), the Susan G. Komen Foundation, the V Foundation for Cancer Research, and Breast Cancer Alliance.

\section{References}

Andreassen, P.R., D'Andrea, A.D., and Taniguchi, T. 2004. ATR couples FANCD2 monoubiquitination to the DNA-damage response. Genes \& Dev. 18: 1958-1963.

Ball, H.L. and Cortez, D. 2005. ATRIP oligomerization is required for ATR-dependent checkpoint signaling. I. Biol. Chem. 280: 31390-31396.

Ball, H.L., Myers, J.S., and Cortez, D. 2005. ATRIP binding to replication protein A-single-stranded DNA promotes ATR-
ATRIP localization but is dispensable for Chk1 phosphorylation. Mol. Biol. Cell 16: 2372-2381.

Bermudez, V.P., Lindsey-Boltz, L.A., Cesare, A.J., Maniwa, Y., Griffith, J.D., Hurwitz, J., and Sancar, A. 2003. Loading of the human 9-1-1 checkpoint complex onto DNA by the checkpoint clamp loader hRad17-replication factor C complex in vitro. Proc. Natl. Acad. Sci. 100: 1633-1638.

Byun, T.S., Pacek, M., Yee, M.C., Walter, J.C., and Cimprich, K.A. 2005. Functional uncoupling of MCM helicase and DNA polymerase activities activates the ATR-dependent checkpoint. Genes \& Dev. 19: 1040-1052.

Chini, C.C. and Chen, J. 2006. Repeated phosphopeptide motifs in human Claspin are phosphorylated by Chk1 and mediate Claspin function. J. Biol. Chem. 281: 33276-33282.

Costanzo, V., Shechter, D., Lupardus, P.J., Cimprich, K.A., Gottesman, M., and Gautier, J. 2003. An ATR- and Cdc7-dependent DNA damage checkpoint that inhibits initiation of DNA replication. Mol. Cell 11: 203-213.

Cuadrado, M., Martinez-Pastor, B., Murga, M., Toledo, L.I., Gutierrez-Martinez, P., Lopez, E., and Fernandez-Capetillo, O. 2006. ATM regulates ATR chromatin loading in response to DNA double-strand breaks. J. Exp. Med. 203: 297-303.

Edwards, R.J., Bentley, N.J., and Carr, A.M. 1999. A Rad3-Rad26 complex responds to DNA damage independently of other checkpoint proteins. Nat. Cell Biol. 1: 393-398.

Ellison, V. and Stillman, B. 2003. Biochemical characterization of DNA damage checkpoint complexes: Clamp loader and clamp complexes with specificity for $5^{\prime}$ recessed DNA. PLOS Biol. 1: E33.

Furuya, K., Poitelea, M., Guo, L., Caspari, T., and Carr, A.M. 2004. Chk1 activation requires Rad9 S/TQ-site phosphorylation to promote association with C-terminal BRCT domains of Rad4TOPBP1. Genes \& Dev. 18: 1154-1164.

Garvik, B., Carson, M., and Hartwell, L. 1995. Single-stranded DNA arising at telomeres in cdc13 mutants may constitute a specific signal for the RAD9 checkpoint. Mol. Cell. Biol. 15: 6128-6138.

Gotter, A.L., Suppa, C., and Emanuel, B.S. 2007. Mammalian TIMELESS and Tipin are evolutionarily conserved replication fork-associated factors. J. Mol. Biol. 366: 36-52.

Hashimoto, Y., Tsujimura, T., Sugino, A., and Takisawa, H. 2006. The phosphorylated C-terminal domain of Xenopus Cut5 directly mediates ATR-dependent activation of Chk1. Genes Cells 11: 993-1007.

Itakura, E., Umeda, K., Sekoguchi, E., Takata, H., Ohsumi, M., and Matsuura, A. 2004. ATR-dependent phosphorylation of ATRIP in response to genotoxic stress. Biochem. Biophys. Res. Commun. 323: 1197-1202.

Itakura, E., Sawada, I., and Matsuura, A. 2005. Dimerization of the ATRIP protein through the coiled-coil motif and its implication to the maintenance of stalled replication forks. Mol. Biol. Cell 16: 5551-5562.

Jazayeri, A., Falck, J., Lukas, C., Bartek, J., Smith, G.C., Lukas, J., and Jackson, S.P. 2006. ATM- and cell cycle-dependent regulation of ATR in response to DNA double-strand breaks. Nat. Cell Biol. 8: 37-45.

Kim, S.M., Kumagai, A., Lee, J., and Dunphy, W.G. 2005. Phosphorylation of Chk1 by ATM- and Rad3-related (ATR) in Xenopus egg extracts requires binding of ATRIP to ATR but not the stable DNA-binding or coiled-coil domains of ATRIP. J. Biol. Chem. 280: 38355-38364.

Kumagai, A. and Dunphy, W.G. 2000. Claspin, a novel protein required for the activation of Chk1 during a DNA replication checkpoint response in Xenopus egg extracts. Mol. Cell 6: 839-849.

Kumagai, A. and Dunphy, W.G. 2003. Repeated phosphopeptide 
motifs in Claspin mediate the regulated binding of Chk1. Nat. Cell Biol. 5: 161-165.

Kumagai, A., Kim, S.M., and Dunphy, W.G. 2004. Claspin and the activated form of ATR-ATRIP collaborate in the activation of Chk1. J. Biol. Chem. 279: 49599-49608.

Kumagai, A., Lee, J., Yoo, H.Y., and Dunphy, W.G. 2006. TopBP1 activates the ATR-ATRIP complex. Cell 124: 943955.

Lee, S.E., Moore, J.K., Holmes, A., Umezu, K., Kolodner, R.D., and Haber, J.E. 1998. Saccharomyces Ku70, mre11/rad50 and RPA proteins regulate adaptation to $\mathrm{G} 2 / \mathrm{M}$ arrest after DNA damage. Cell 94: 399-409.

Lee, J., Kumagai, A., and Dunphy, W.G. 2003. Claspin, a Chk1regulatory protein, monitors DNA replication on chromatin independently of RPA, ATR, and Rad17. Mol. Cell 11: 329340.

Leung-Pineda, V., Ryan, C.E., and Piwnica-Worms, H. 2006. Phosphorylation of Chk1 by ATR is antagonized by a Chk1regulated protein phosphatase $2 \mathrm{~A}$ circuit. Mol. Cell. Biol. 26: 7529-7538.

Lopes, M., Foiani, M., and Sogo, J.M. 2006. Multiple mechanisms control chromosome integrity after replication fork uncoupling and restart at irreparable UV lesions. Mol. Cell 21: 15-27.

Lucca, C., Vanoli, F., Cotta-Ramusino, C., Pellicioli, A., Liberi, G., Haber, J., and Foiani, M. 2004. Checkpoint-mediated control of replisome-fork association and signalling in response to replication pausing. Oncogene 23: 1206-1213.

Lupardus, P.J. and Cimprich, K.A. 2006. Phosphorylation of Xenopus Rad1 and Hus1 defines a readout for ATR activation that is independent of Claspin and the Rad9 carboxy terminus. Mol. Biol. Cell 17: 1559-1569.

Lupardus, P.J., Byun, T., Yee, M.C., Hekmat-Nejad, M., and Cimprich, K.A. 2002. A requirement for replication in activation of the ATR-dependent DNA damage checkpoint. Genes \& Dev. 16: 2327-2332.

MacDougall, C.A., Byun, T.S., Van, C., Yee, M., and Cimprich, K.A. 2007. The structural determinants of checkpoint activation. Genes \& Dev. (this issue).

Majka, J. and Burgers, P.M. 2003. Yeast Rad17/Mec3/Ddc1: A sliding clamp for the DNA damage checkpoint. Proc. Natl. Acad. Sci. 100: 2249-2254.

Majka, J., Binz, S.K., Wold, M.S., and Burgers, P.M. 2006a. Replication protein A directs loading of the DNA damage checkpoint clamp to $5^{\prime}$-DNA junctions. J. Biol. Chem. 281: 2785527861.

Majka, J., Niedziela-Majka, A., and Burgers, P.M. 2006b. The checkpoint clamp activates Mec1 kinase during initiation of the DNA damage checkpoint. Mol. Cell 24: 891-901.

Michael, W.M., Ott, R., Fanning, E., and Newport, J. 2000. Activation of the DNA replication checkpoint through RNA synthesis by primase. Science 289: 2133-2137.

Myers, J.S. and Cortez, D. 2006. Rapid activation of ATR by ionizing radiation requires ATM and Mre11. J. Biol. Chem. 281: 9346-9350.

Nakada, D., Hirano, Y., and Sugimoto, K. 2004. Requirement of the Mre11 complex and exonuclease 1 for activation of the Mec1 signaling pathway. Mol. Cell. Biol. 24: 10016-10025.

Nakada, D., Hirano, Y., Tanaka, Y., and Sugimoto, K. 2005. Role of the C terminus of Mec1 checkpoint kinase in its localization to sites of DNA damage. Mol. Biol. Cell 16: 5227-5235.

Namiki, Y. and Zou, L. 2006. ATRIP associates with replication protein A-coated ssDNA through multiple interactions. Proc. Natl. Acad. Sci. 103: 580-585.

Osborn, A.J. and Elledge, S.J. 2003. Mrc1 is a replication fork component whose phosphorylation in response to DNA rep- lication stress activates Rad53. Genes \& Dev. 17: 17551767.

Pacek, M. and Walter, J.C. 2004. A requirement for MCM7 and Cdc45 in chromosome unwinding during eukaryotic DNA replication. EMBO J. 23: 3667-3676.

Paciotti, V., Clerici, M., Lucchini, G., and Longhese, M.P. 2000. The checkpoint protein Ddc2, functionally related to $S$. pombe Rad26, interacts with Mecl and is regulated by Mec1-dependent phosphorylation in budding yeast. Genes \& Dev. 14: 2046-2059.

Sogo, J.M., Lopes, M., and Foiani, M. 2002. Fork reversal and ssDNA accumulation at stalled replication forks owing to checkpoint defects. Science 297: 599-602.

St Onge, R.P., Besley, B.D., Pelley, J.L., and Davey, S. 2003. A role for the phosphorylation of hRad9 in checkpoint signaling. J. Biol. Chem. 278: 26620-26628.

Stokes, M.P., Van Hatten, R., Lindsay, H.D., and Michael, W.M. 2002. DNA replication is required for the checkpoint response to damaged DNA in Xenopus egg extracts. I. Cell Biol. 158: 863-872.

Unsal-Kacmaz, K., Chastain, P.D., Qu, P.P., Minoo, P., Cordeiro-Stone, M., Sancar, A., and Kaufmann, W.K. 2007. The human Tim/Tipin complex coordinates an intra-S checkpoint response to UV that slows replication fork displacement. Mol. Cell. Biol. [Epub 12 February 2007; DOI: 10.1128/MCB.02190-06]

Vaze, M.B., Pellicioli, A., Lee, S.E., Ira, G., Liberi, G., ArbelEden, A., Foiani, M., and Haber, J.E. 2002. Recovery from checkpoint-mediated arrest after repair of a double-strand break requires Srs2 helicase. Mol. Cell 10: 373-385.

Wang, H. and Elledge, S.J. 2002. Genetic and physical interactions between DPB11 and DDC1 in the yeast DNA damage response pathway. Genetics 160: 1295-1304.

Wang, Y. and Qin, J. 2003. MSH2 and ATR form a signaling module and regulate two branches of the damage response to DNA methylation. Proc. Natl. Acad. Sci. 100: 15387-15392.

Wang, X., Zou, L., Lu, T., Bao, S., Hurov, K.E., Hittelman, W.N., Elledge, S.J., and Li, L. 2006. Rad17 phosphorylation is required for claspin recruitment and Chk1 activation in response to replication stress. Mol. Cell 23: 331-341.

Ward, I.M. and Chen, J. 2001. Histone H2AX is phosphorylated in an ATR-dependent manner in response to replicational stress. J. Biol. Chem. 276: 47759-47762.

Yoo, H.Y., Shevchenko, A., Shevchenko, A., and Dunphy, W.G. 2004. Mcm2 is a direct substrate of ATM and ATR during DNA damage and DNA replication checkpoint responses. J. Biol. Chem. 279: 53353-53364.

Yoo, H.Y., Jeong, S.Y., and Dunphy, W.G. 2006. Site-specific phosphorylation of a checkpoint mediator protein controls its responses to different DNA structures. Genes \& Dev. 20: 772-783.

You, Z., Kong, L., and Newport, J. 2002. The role of singlestranded DNA and polymerase $\alpha$ in establishing the ATR, Hus1 DNA replication checkpoint. J. Biol. Chem. 277: 27088-27093.

Zhao, H., Tanaka, K., Nogochi, E., Nogochi, C., and Russell, P. 2003. Replication checkpoint protein $\mathrm{Mrcl}$ is regulated by Rad3 and Tell in fission yeast. Mol. Cell. Biol. 23: 83958403.

Zou, L. and Elledge, S.J. 2003. Sensing DNA damage through ATRIP recognition of RPA-ssDNA complexes. Science 300: $1542-1548$.

Zou, L., Liu, D., and Elledge, S.J. 2003. Replication protein Amediated recruitment and activation of Rad17 complexes. Proc. Nat1. Acad. Sci. 100: 13827-13832. 


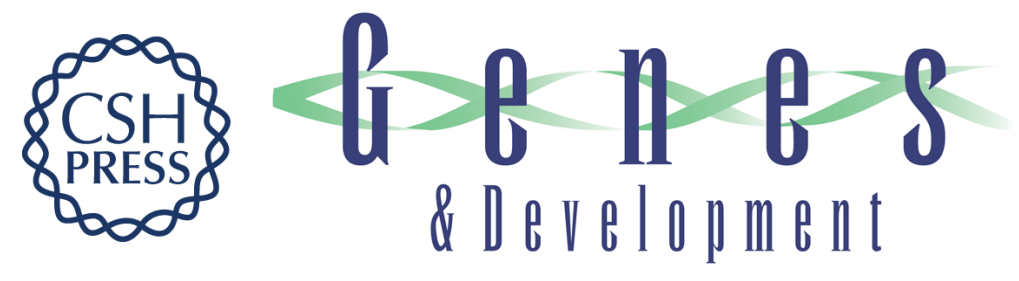

\section{Single- and double-stranded DNA: building a trigger of ATR-mediated DNA damage response}

Lee Zou

Genes Dev. 2007, 21:

Access the most recent version at doi:10.1101/gad.1550307
Related Content The structural determinants of checkpoint activation
Christina A. MacDougall, Tony S. Byun, Christopher Van, et al.
Genes Dev. April , 2007 21: 898-903
References This article cites 54 articles, 37 of which can be accessed free at:
http://genesdev.cshlp.org/content/21/8/879.full.html\#ref-list-1
Articles cited in:
http://genesdev.cshlp.org/content/21/8/879.full.html\#related-urls
License
Email Alerting Receive free email alerts when new articles cite this article - sign up in the box at the top Service right corner of the article or click here.

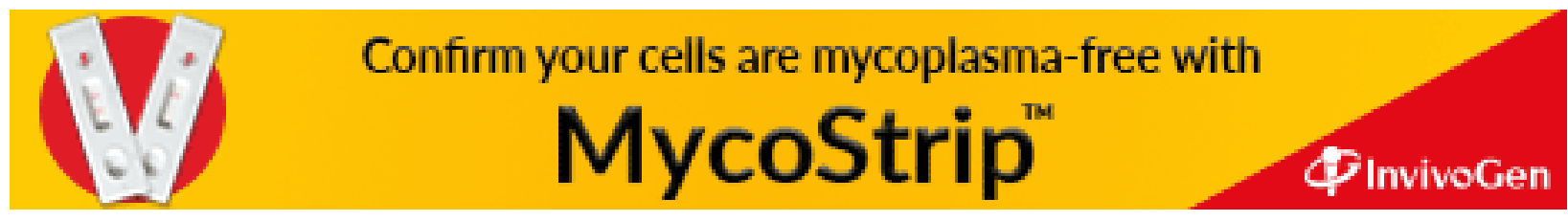

